\title{
A globally convergent generalized pole-placement adaptive control algorithm
}

\author{
WEI WANG $\dagger$ and ROLF HENRIKSEN $\ddagger$
}

Keywords: Predictive control, adaptive control, pole-placement, convergence, stability.

A complete proof of global convergence of a generalized pole-placement adaptive control algorithm for linear discrete-time plants with an unmeasured bounded disturbance is given in this paper. The analysis shows that the difficulty encountered in the convergence analysis is circumvented by fixing the parameters which enter nonlinearly into the control law to some constant value. This allows us to use the standard analysis techniques for adaptive control in the analysis of our control algorithm.

\section{Introduction}

Adaptive control based on long-range predictive methods has evoked a great deal of interest recently, see, for instance, extended horizon adaptive control (EHAC) (Ydstie 1984), extended prediction self-adaptive control (EPSAC) (De Keyser and Van Cauwenberghe 1985), generalized predictive control (GPC) (Clarke et al. 1987), and generalized pole-placement control (GPP) (Lelic and Zarrop 1987).

In spite of the intense research efforts, the question of convergence of adaptive controllers based on long-range predictive methods is still open. This type of control law is generally nonlinear in the estimated parameters which causes a great difficulty in the convergence analysis. Ortega and Sanchez (1989) pointed out that to date it is not clear how to establish the linear boundedness condition of the key technical lemma (Goodwin and Sin 1984) for a control structure which is non-linear in parameters.

To circumvent this difficulty, Ortega and Sanchez (1989) used a fairly restrictive assumption that the first $N$ coefficients of the plant impulse response are known and suggested a globally convergent multistep receding horizon adaptive controller (MRHAC) for the deterministic plant. Although the non-linear control law can be changed into a linear one in this way, this assumption reduces the significance of the result since, for general plants, it is difficult to determine these coefficients a priori when sufficient knowledge regarding the plant is absent. In addition, the parameters of the controller are estimated on-line in the MRHAC algorithm and a bank of estimators for unknown parameters must be used.

Received 5 August 1991.

$\dagger$ Department of Automatic Control, Northeast University of Technology, Shenyang, 110006, P.R. China. Currently on leave at Division of Engineering Cybernetics, the Norwegian Institute of Technology, N-7034 Trondheim, Norway.

$\ddagger$ Division of Engineering Cybernetics, the Norwegian Institute of Technology, N-7034 Trondheim, Norway.

This paper was presented at the European Control Conference ECC '91, 2-5 July 1991, Grenoble, France, and is reprinted with permission. 
In this paper a globally convergent adaptive control algorithm based on the GPP method (Lelic and Zarrop 1987) is proposed which provides a way to establish the convergence of this type of adaptive controllers. In the proposed adaptive control algorithm the non-linear parameter of the control law are not updated on-line, instead of that they are fixed to some constant value. The restrictive assumption about the coefficients of the plant impulse response in Ortega and Sanchez (1989) is, however, not needed. We will deal with linear time-invariant discrete-time deterministic plants with an unmeasured bounded disturbance. As will become clear in the sequel the convergence and stability results can be extended to treat the case of stochastic disturbances via martingale theory.

\section{Generalized Pole-Placement (GPP) scheme}

In this section the GPP scheme is introduced briefly with a trivial modification (see Lelic and Zarrop 1987 for further details).

The plant to be controlled is of discrete-time with constant but unknown parameters and is described by the difference equation

$$
A\left(q^{-1}\right) y(t)=B\left(q^{-1}\right) u(t)+\xi(t)
$$

where $A$ and $B$ are polynomials in the backward shift operator $q^{-1}$ of the form

$$
\begin{gathered}
A\left(q^{-1}\right)=1+a_{1} q^{-1}+\ldots+a_{n} q^{-n} \\
B\left(q^{-1}\right)=q^{-d} B^{\prime}\left(q^{-1}\right)=q^{-d}\left(b_{0}+b_{1} q^{-1}+\ldots+b_{m} q^{-m}\right)
\end{gathered}
$$

$u(t)$ and $y(t)$ are the plant input and output respectively, $d \geqslant 1$ and $b_{0} \neq 0$. $\xi(t)$ is an unmeasured bounded disturbance. Let $M$ be an upper bound of $|\xi(t)|$.

The following assumptions are made about the plant (2.1).

A1: $n$ and $m$ are known.

$\mathrm{A} 2: A\left(q^{-1}\right)$ and $B\left(q^{-1}\right)$ are coprime.

A3: $d$ is known.

A4: $M$ is known.

Define a generalized output

$$
\phi_{j}(t)=P_{j} y(t+d+j-1)-R y_{r}(t+d+j-1)+Q u(t-1)
$$

where

$$
\begin{gathered}
P_{j}\left(q^{-1}\right)=p_{0}+p_{1} q^{-1}+\ldots+p_{j+d-2} q^{-j-d+2} \\
Q=Q_{n} / Q_{d}, \quad Q_{d}(0)=1
\end{gathered}
$$

where $R, Q_{n}$ and $Q_{d}$ are polynomials in $q^{-1} .\left\{y_{r}(t)\right\}$ is a bounded reference sequence. Note that the polynomial $P_{j}\left(q^{-1}\right)$ is different from the one used in Lelic and Zarrop (1987) and its use will be seen in the adaptive case.

Use of the following polynomial equations

$$
\begin{gathered}
P_{j}=A F_{j}+q^{-j-d+1} G_{j} \\
B^{\prime} F_{j}=E_{j}+q^{-j} \Gamma_{j}
\end{gathered}
$$


where

$$
\begin{gathered}
F_{j}\left(q^{-1}\right)=f_{0}+f_{1} q^{-1}+\ldots+f_{j+d-2} q^{-j-d+2} \\
E_{j}\left(q^{-1}\right)=e_{0}+e_{1} q^{-1}+\ldots+e_{j-1} q^{-j+1} \\
G_{j}\left(q^{-1}\right)=g_{0}^{j}+g_{1}^{j} q^{-1}+\ldots+g_{n-1}^{j} q^{-n+1} \\
\Gamma_{j}\left(q^{-1}\right)=\gamma_{0}^{j}+\gamma_{1}^{j} q^{-1}+\ldots+\gamma_{m+d-2}^{j} q^{-m-d+2}
\end{gathered}
$$

leads via a standard manipulation to

$\phi_{j}(t)=E_{j} u(t+j-1)+\left(\Gamma_{j}+Q\right) u(t-1)+G_{j} y(t)-R y_{r}(t+d+j-1)+F_{j} \xi(t+d+j-1)$

where $j=1, \ldots N$.

The equation (2.8) can be written in the vector form

$$
\Phi=E u+\psi u(t-1)+G y(t)-R w+F \xi
$$

where

$$
\begin{gathered}
\Phi^{\mathrm{T}}=\left[\phi_{1}(t) \ldots \phi_{N}(t)\right] \\
u^{\mathrm{T}}=[u(t) \ldots u(t+N-1)] \\
w^{\mathrm{T}}=\left[y_{r}(t+d) \ldots y_{r}(t+d+N-1)\right] \\
\xi^{\mathrm{T}}=[\xi(t+d) \ldots \xi(t+d+N-1)] \\
\psi^{\mathrm{T}}=\left[\left(\Gamma_{1}+Q\right) \ldots\left(\Gamma_{N}+Q\right)\right] \\
G^{\mathrm{T}}=\left[G_{1} \ldots G_{N}\right] \\
F^{\mathrm{T}}=\left[F_{1} \ldots F_{N}\right]
\end{gathered}
$$

and where $E$ is the $N \times N$ lower-triangular matrix

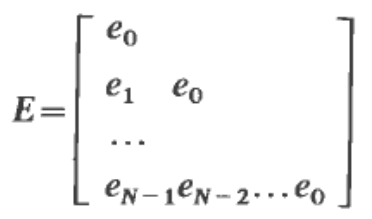

The current control $u(t)$ is given by

$$
u(t)=K^{\mathrm{T}}[R w-\psi u(t-1)-G y(t)]
$$

where

$$
K^{\mathbf{T}}=\left[k_{1} \ldots k_{N}\right]
$$

is the first row of $\left(E^{\mathrm{T}} E+\lambda I\right)^{-1} E^{\mathrm{T}}$.

Remark 2.1. We know from Lelic and Zarrop (1987) that $u(t)$ given in (2.11) is the 'rolling horizon' control law if $\{\xi(t)\}$ is a white noise sequence and the following cost function is used

$$
J=\mathbf{E}\left(\Phi^{\mathrm{T}} \Phi+\lambda u^{\mathrm{T}} u\right)
$$

where $\mathrm{E}($.$) is the expectation operator.$

Using (2.4) and the definition of $\psi$, equation (2.11) takes the form

$$
L u(t)+Q_{d} K^{\mathrm{T}} G y(t)=Q_{d} R K_{1} y_{r}(t+d+N-1)
$$


where

$$
\begin{gathered}
K_{1}=k_{N}+k_{N-1} q^{-1}+\ldots+k_{1} q^{-N+1} \\
L=Q_{d}+q^{-1} Q_{d} K^{\mathrm{T}} \Gamma+q^{-1} \mu Q_{n} \\
\Gamma^{\mathrm{T}}=\left[\Gamma_{1} \ldots \Gamma_{N}\right] \\
\mu=\sum_{j=1}^{N} k_{j}
\end{gathered}
$$

Combining (2.13) and (2.1) we obtain the closed-loop system

$$
\left(A_{1} Q_{d}+B_{1} Q_{n}\right) y(t)=B^{\prime} Q_{d} R K_{1} y_{r}(t+N-1)+L \xi(t)
$$

where

$$
\begin{gathered}
A_{1}=A+q^{-1} A K^{\mathrm{T}} \Gamma+q^{-d} B^{\prime} K^{\mathrm{T}} G \\
B_{1}=q^{-1} \mu A
\end{gathered}
$$

From (2.5) and (2.6) we have

$$
B^{\prime} P_{j}-A E_{j}=q^{-j+1}\left(q^{-d} B^{\prime} G_{j}+q^{-1} A \Gamma_{j}\right)
$$

Define

$$
D_{j}=q^{-d} B^{\prime} G_{j}+q^{-1} A \Gamma_{j}
$$

Thus (2.16) takes the form

$$
A_{1}=A+K^{\mathrm{T}} D
$$

where

$$
D^{\mathrm{T}}=\left[D_{1} \ldots D_{N}\right]
$$

From (2.17) and (2.18)-(2.20) we know that $\operatorname{deg} B_{1}=n+1$ and $\operatorname{deg} A_{1}=\operatorname{deg} D_{j}=\max (m$ $+d, n)$.

To assign the closed-loop poles, select $Q_{n}$ and $Q_{d}$ such that

$$
A_{1} Q_{d}+B_{1} Q_{n}=T
$$

where $T\left(q^{-1}\right)$ is a prespecified stable polynomial and the zeros of $T\left(q^{-1}\right)$ are the required poles. It is then possible to ensure the stability of the closed-loop system with the GPP controller. There exists a unique solution of (2.21) for the coefficient of $Q_{n}$ and $Q_{d}$ if their degrees satisfy $\operatorname{deg} T \leqslant \operatorname{deg} A_{1}+\operatorname{deg} B_{1}-1, \operatorname{deg} Q_{n}=\operatorname{deg} A_{1}-1$ and $\operatorname{deg} Q_{d}$ $=\operatorname{deg} B_{1}-1$.

For removing steady-state output tracking error in (2.15) the polynomial $R\left(q^{-1}\right)$ must satisfy

$$
R(1)=\frac{T(1)}{\mu B(1) Q_{d}(1)}
$$

In order to avoid singularity of $\left(E^{\mathrm{T}} E+\lambda I\right)$ and to reduce the computational burden for large output horizons, the technique of imposing a control horizon $N_{u}$ can be used here, see Lelic and Zarrop (1987) for details. 
3. Adaptive control algorithm and convergence analysis

Let us rewrite (2.1) as follows

where

$$
y(t)=X(t-1)^{\mathrm{T}} \theta+\xi(t)
$$

$$
\begin{gathered}
X(t-1)^{\mathrm{T}}=[-y(t-1) \ldots-y(t-n), u(t-d) \ldots u(t-d-m)] \\
\theta^{\mathrm{T}}=\left[a_{1} \ldots a_{m}, b_{0} \ldots b_{m}\right]
\end{gathered}
$$

When the plant parameter vector $\theta$ in (3.1) is unknown we use the estimate $\theta(t)$ instead of $\theta$. Define

$$
\begin{gathered}
\hat{\theta}(t)^{\mathrm{T}}=\left[\hat{a}_{1}(t), \ldots, \hat{a}_{n}(t), \hat{b}_{0}(t), \ldots, \hat{b}_{m}(t)\right] \\
\varepsilon(t)=y(t)-X(t-1)^{\mathrm{T}} \hat{\theta}(t-1)
\end{gathered}
$$

The following recursive least-squares parameter estimation with a dead zone is used here:

$$
\begin{gathered}
\hat{\theta}(t)=\hat{\theta}(t-1)+\frac{\alpha(t) P(t-2) X(t-1) \varepsilon(t)}{1+X(t-1)^{\mathrm{T}} P(t-2) X(t-1)} \\
P(t)=P(t-1)-\frac{\alpha(t+1) P(t-1) X(t) X(t)^{\mathrm{T}} P(t-1)}{1+X(t)^{\mathrm{T}} P(t-1) X(t)} \\
\alpha(t)= \begin{cases}0 & \text { if }|\varepsilon(t)|<2 M \\
\eta & \text { otherwise }(\eta \in(\omega, 3 / 4(1-\omega)), 0<\omega<3 / 7)\end{cases}
\end{gathered}
$$

Lemma 3.1. The estimation scheme (3.3)-(3.5) has the following properties:

(i) $v(t)-v(t-1) \leqslant 0, \quad$ where $v(t)=\tilde{\theta}(t)^{\mathrm{T}} P(t-1)^{-1} \tilde{\theta}(t), \tilde{\theta}(t)=\theta(t)-\theta$.

(ii) $\lim _{t \rightarrow \infty} \frac{\alpha(t) \varepsilon(t)^{2}}{1+X(t-1)^{\mathrm{T}} P(t-2) X(t-1)}=0$.

(iii) $\lim _{t \rightarrow \infty}\|\hat{\theta}(t)-\hat{\theta}(t-k)\|=0, \quad$ for any finite $k$.

Proof. See Gu and Wang (1989).

At first glance it would seem reasonable to obtain an adaptive control algorithm by combining the control law (2.11) and the estimation scheme (3.3)-(3.5) and using the certainty equivalence principle. But the convergence of this adaptive control algorithm can generally not be ensured, and its analysis is very difficult since the control law (2.11) is non-linear in the estimated parameters $e_{j}(j=0,1, \ldots, N-1)$. From equation (2.11) we know that the non linearity in the control law is due to the fact that the $e_{j}$ s are updated on-line. However, if they are fixed to some prespecified constant value, the difficulty encountered in the convergence analysis can be overcome. We therefore propose the following adaptive control algorithm.

Data: the polynomial $T\left(q^{-1}\right)$, the horizons $N$ and $N_{u}$, the coefficient $\lambda$ and $e_{j}$ $(j=0,1, \ldots, N-1)$.

(a) Estimate the parameters of plant (2.1) by using (3.3)-(3.5).

(b) Use the estimate $\hat{\theta}(t)$ to form $\hat{A}\left(t, q^{-1}\right)$ and $\hat{B}^{\prime}\left(t, q^{-1}\right)$, and compute $\hat{\Gamma}_{j}, \hat{F}_{j}$ and $\hat{G}_{j}$ using the following polynomial equations

$$
\hat{B}^{\prime}\left(t, q^{-1}\right) \hat{F}_{j}\left(t, q^{-1}\right)=E_{j}\left(q^{-1}\right)+q^{-j} \hat{\Gamma}_{j}\left(t, q^{-1}\right)
$$




$$
\hat{P}_{j}\left(t, q^{-1}\right)=\hat{A}\left(t, q^{-1}\right) \hat{F}_{j}\left(t, q^{-1}\right)+q^{-j-d+1} \hat{G}_{j}\left(t, q^{-1}\right)
$$

where

$$
\begin{aligned}
& \hat{A}\left(t, q^{-1}\right)=1+\hat{\theta}_{1}(t) q^{-1}+\ldots+\hat{\theta}_{n}(t) q^{-n} \\
& \hat{B}^{\prime}\left(t, q^{-1}\right)=\hat{\theta}_{n+1}(t)+\hat{\theta}_{n+2}(t) q^{-1}+\ldots+\hat{\theta}_{n+m+1}(t) q^{-m} \\
& \hat{P}_{j}\left(t, q^{-1}\right)=\hat{p}_{0}(t)+\hat{p}_{1}(t) q^{-1}+\ldots+\hat{p}_{j+d-2}(t) q^{-j-d+2}
\end{aligned}
$$

(c) Solve the diophantine equation for $\hat{Q}_{d}$ and $\hat{Q}_{n}$ using

$$
\hat{A}_{1}\left(t, q^{-1}\right) \hat{Q}_{d}\left(t, q^{-1}\right)+\hat{B}_{1}\left(t, q^{-1}\right) \hat{Q}_{n}\left(t, q^{-1}\right)=T\left(q^{-1}\right)
$$

where $T\left(q^{-1}\right)$ is a prespecified stable polynomial and

$$
\begin{gathered}
\hat{A}_{1}\left(t, q^{-1}\right)=\hat{A}\left(t, q^{-1}\right)+K^{\mathrm{T}} \hat{D}\left(t, q^{-1}\right) \\
\hat{B}_{1}\left(t, q^{-1}\right)=q^{-1} \mu \hat{A}\left(t, q^{-1}\right)
\end{gathered}
$$

where

$$
\begin{gathered}
\hat{D}^{\mathrm{T}}\left(t, q^{-1}\right)=\left[\hat{D}_{1}\left(t, q^{-1}\right), \ldots, \hat{D}_{N}\left(t, q^{-1}\right)\right] \\
\hat{D}_{j}\left(t, q^{-1}\right)=q^{-d} \hat{B}^{\prime}\left(t, q^{-1}\right) \hat{G}_{j}\left(t, q^{-1}\right)+q^{-1} \hat{A}\left(t, q^{-1}\right) \hat{\Gamma}_{j}\left(t, q^{-1}\right)
\end{gathered}
$$

(d) The control $u(t)$ is then determined from

$$
\hat{L}\left(t, q^{-1}\right) u(t)+\hat{G}_{0}\left(t, q^{-1}\right) y(t)=\hat{R}_{0}\left(t, q^{-1}\right) y_{r}(t+d+N-1)
$$

where

$$
\begin{gathered}
\hat{L}\left(t, q^{-1}\right)=\hat{Q}_{d}\left(t, q^{-1}\right)+q^{-1} \hat{Q}_{d}\left(t, q^{-1}\right) K^{\mathrm{T}} \hat{\Gamma}\left(t, q^{-1}\right)+q^{-1} \mu \hat{Q}_{n}\left(t, q^{-1}\right) \\
\hat{G}_{0}\left(t, q^{-1}\right)=\hat{Q}_{d}\left(t, q^{-1}\right) K^{\mathrm{T}} \hat{G}\left(t, q^{-1}\right) \\
\hat{R}_{0}\left(t, q^{-1}\right)=\hat{Q}_{d}\left(t, q^{-1}\right) \hat{R}\left(t, q^{-1}\right) K_{1}\left(q^{-1}\right) \\
\hat{R}(1)=\frac{T(1)}{\mu \hat{B}(1) \hat{Q}_{d}(1)}
\end{gathered}
$$

Remark 3.1. We know from (3.6) that $\hat{\theta}_{n+1}(t)=\hat{b}_{0}(t) \neq 0$ is required to ensure the solvability for $\hat{F}_{j}, \hat{\Gamma}_{j}, \hat{P}_{j}$ and $\hat{G}_{j}$. For this purpose the gain $\eta$ in the estimation algorithm can be adjusted to ensure that $\theta_{n+1}(t)$ does not go to zero.

Remark 3.2. In comparison with the GPP algorithm in Lelic and Zarrop (1987) our algorithm needs values of $e_{j}(j=0, \ldots, N-1)$ in the initiation in addition to other data. This is, however, not difficult. If the prior knowledge of the impulse response coefficients $\delta_{j}(j=0,1, \ldots, N-1)$ of the plant are known, $e_{j}=\delta_{j}(j=0, \ldots, N-1)$ are recommended. Some simulations have showed that the choice of $e_{j}$ is not critical and sensitive. It is also clear from (2.12) and (2.14) that $K$ and $\mu$ can be calculated off-line if the parameters $\lambda$ and $e_{j}(j=0, \ldots, N-1)$ are given.

From this algorithm we know that the convergence depends heavily on the solvability of equation (3.9). Although (3.9) is solvable with probability one (Goodwin and $\operatorname{Sin} 1981$ ), in the following analysis we require $\hat{Q}_{d}$ and $\hat{Q}_{n}$ to have bounded coefficients and hence near-singularity of (3.9) must also be avoided. It follows from $\mathrm{Gu}$ and Wang (1988) that the singularity of the time-varying equation (3.9) can be avoided by the use of the estimation algorithm (3.3)-(3.5). 
Theorem 3.1. Subject to assumptions A1-A4 the adaptive control algorithm given by $(a)(d)$, when applied to plant (2.1), leads to

(i) $\{y(t)\}$ and $\{u(t)\}$ are bounded for all $t$.

(ii) there exists a $T_{1}>0$ such that for $t \geqslant T_{1}$

$$
|\hat{A} y(t)-\hat{B} u(t)|<2 M
$$

Proof. Given the time varying polynomials $\hat{A}\left(t, q^{-1}\right)$ and $\hat{B}\left(t, q^{-1}\right)$, define

$$
\begin{gathered}
\hat{A} \hat{B}=\hat{A}\left(t, q^{-1}\right) \hat{B}\left(t, q^{-1}\right)=\sum_{i} \sum_{j} \hat{a}_{i}(t) b_{j}(t) q^{-i-j}=\hat{B} \hat{A} \\
\hat{A} \cdot \hat{B}=\hat{A}\left(t, q^{-1}\right) \cdot \hat{B}\left(t, q^{-1}\right)=\sum_{i} \sum_{j} \hat{a}_{i}(t) b_{j}(t-i) q^{-i-j} \neq \hat{B} \cdot \hat{A} \\
\bar{A}=\hat{A}\left(t-1, q^{-1}\right), \quad \bar{B}=\hat{B}\left(t-1, q^{-1}\right)
\end{gathered}
$$

From (3.2) and (3.19) we have

$$
\varepsilon(t)=\bar{A} y(t)-\bar{B} u(t)
$$

Multiplying (3.20) by $\hat{G}_{0}$ and $\hat{L}$ respectively we have

$$
\begin{aligned}
\hat{G}_{0} \varepsilon(t) & =\hat{G}_{0} \cdot \bar{A} y(t)-\hat{G}_{0} \cdot \bar{B} u(t) \\
& =\hat{G}_{0} \hat{A} y(t)-\hat{G}_{0} B u(t)+\left[\hat{G}_{0} \cdot \bar{A}-\hat{G}_{0} \hat{A}\right] y(t)-\left[\hat{G}_{0} \cdot \hat{B}-\hat{G}_{0} \hat{B}\right] u(t)
\end{aligned}
$$

and

$$
\begin{aligned}
\hat{L} \varepsilon(t) & =\hat{L} \cdot \bar{A} y(t)-\hat{L} \cdot \bar{B} u(t) \\
& =\hat{L} \hat{A} y(t)-\hat{L} \hat{B} u(t)+[\hat{L} \cdot \bar{A}-\hat{L} \hat{A}] y(t)-[\hat{L} \cdot \bar{B}-\hat{L} \hat{B}] u(t)
\end{aligned}
$$

Now, define

$$
w(t)=\bar{A} \cdot \hat{R}_{0} y_{r}(t+d+N-1)
$$

Using (3.9) $-(3.17)$ and (3.21) we have

$$
\begin{aligned}
w(t)= & \bar{A} \cdot \hat{L} u(t)+\bar{A} \cdot \hat{G}_{0} y(t) \\
= & \hat{A} \hat{L} u(t)+\hat{A} \hat{G}_{0} y(t)+[\bar{A} \cdot \hat{L}-\hat{A} \hat{L}] u(t)+\left[\bar{A} \cdot \hat{G}_{0}-\hat{A} \hat{G}_{0}\right] y(t) \\
= & \hat{A} \hat{L} u(t)+\hat{G}_{0} \varepsilon(t)+\hat{G}_{0} \hat{B} u(t)+[\bar{A} \cdot \hat{L}-\hat{A} \hat{L}] u(t)+\left[\bar{A} \cdot \hat{G}_{0}-\hat{A} \hat{G}_{0}\right] y(t) \\
& +\left[\hat{G}_{0} \cdot \bar{B}-\hat{G}_{0} \hat{B}\right] u(t)-\left[\hat{G}_{0} \cdot \bar{A}-\hat{G}_{0} \hat{A}\right] y(t) \\
= & \left(T+\Delta_{1}\right) u(t)+\hat{G}_{0} \varepsilon(t)+\Delta_{2} y(t)
\end{aligned}
$$

where

$$
\begin{gathered}
\Delta_{1}=\left[\hat{G}_{0} \cdot \bar{B}-\hat{G}_{0} \hat{B}\right]+[\bar{A} \cdot \hat{L}-\hat{A} \hat{L}] \\
\Delta_{2}=\left[\bar{A} \cdot \hat{G}_{0}-\hat{A} \hat{G}_{0}\right]-\left[\hat{G}_{0} \cdot \bar{A}-\hat{G}_{0} \hat{A}\right]
\end{gathered}
$$

Also, define

$$
z(t)=\bar{B} \cdot \hat{R}_{0} y_{\mathrm{r}}(t+d+N-1)
$$

Using a similar argument to the latter we obtain

$$
\begin{aligned}
z(t) & =\bar{B} \cdot \hat{L} u(t)+\bar{B} \cdot \hat{G}_{0} y(t) \\
& =\left(T+\Delta_{4}\right) y(t)-\hat{L} \varepsilon(t)+\Delta_{3} u(t)
\end{aligned}
$$


where

$$
\begin{gathered}
\Delta_{3}=[\bar{B} \cdot \hat{L}-\hat{B} \hat{L}]-[\hat{L} \cdot \bar{B}-\hat{L} \hat{B}] \\
\Delta_{4}=[\hat{L} \cdot \bar{A}-\hat{L} \hat{A}]+\left[\bar{B} \cdot \hat{G}_{0}-\hat{B} \hat{G}_{0}\right]
\end{gathered}
$$

Combining (3.23) and (3.24) and from the definition of $w(t)$ and $z(t)$ we have

$$
\left[\begin{array}{cc}
T+\Delta_{1} & \Delta_{2} \\
\Delta_{3} & T+\Delta_{4}
\end{array}\right]\left[\begin{array}{c}
u(t) \\
y(t)
\end{array}\right]=\left[\begin{array}{c}
\left(\hat{A} \hat{R}_{0}+\left[\bar{A} \cdot \hat{R}_{0}-\hat{A} \hat{R}_{0}\right]\right) y_{r}(t+d+N-1)-\hat{G}_{0} \varepsilon(t) \\
\left(\hat{B} R_{0}+\left[\bar{B} \cdot \hat{R}_{0}-\hat{B} R_{0}\right]\right) y_{r}(t+d+N-1)+\hat{L} \varepsilon(t)
\end{array}\right]
$$

Equation (3.25) has a similar structure as (3.37) in Goodwin and Sin (1981). It can be regarded as a linear time varying dynamical system with inputs $\left\{y_{r}(t)\right\}$ and $\{\varepsilon(t)\}$ and outputs $\{u(t)\}$ and $\{y(t)\}$. Now, it follows from Gu and Wang (1988) that $\hat{A}$ and $\hat{B}$ are always coprime which means, from (3.10)-(3.13), that $\hat{A}_{1}$ and $\hat{B}_{1}$ must be coprime and have bounded coefficients. Therefore $\hat{Q}_{d}$ and $\hat{Q}_{n}$ must have bounded coefficients for all $t$. From lemma 3.1 (iii) and the continuity of (3.9), it follows that $\Delta_{1}, \Delta_{2}, \Delta_{3}, \Delta_{4},\left[\bar{A} \cdot \hat{R}_{0}\right.$ $\left.-\hat{A} \hat{R}_{0}\right]$ and $\left[\bar{B} \cdot \hat{R}_{0}-\hat{B} \hat{R}_{0}\right]$ in (3.25) all approach zero as $t$ tends to infinity. Then, for a sufficiently large but finite $t$, the system (3.25) is arbitrarily close to an asymptotically exponentially stable system having characteristic polynomial $T(z)^{2}$. From (3.25) and the boundedness of $\left\{y_{r}(t)\right\}$ it follows that the sequences $\{u(t)\}$ and $\{y(t)\}$, and hence $\{\|X(t)\|\}$ will not grow faster than linearly with respect to $\varepsilon(t)$. Then, from lemma 3.1 (ii) we can apply the key technical lemma (Goodwin and Sin 1984) to conclude that

$$
\lim _{t \rightarrow \infty} \alpha(t) \varepsilon(t)^{2}=0
$$

and $\{\|X(t)\|\}$ must be bounded. This establishes part (i) of the theorem.

Define $\mathbf{H}=\left\{t: \alpha(t) \neq 0, t \in N^{+}\right\}$. We first prove that $\mathrm{H}$ must be a finite set. If not, a series $\left\{t_{n}\right\}$ can be obtained where $t_{n} \rightarrow \infty$ as $n \rightarrow \infty$. From (3.26) it follows readily that

$$
\lim _{t \rightarrow \infty} \varepsilon\left(t_{n}\right)=0
$$

Then, for a sufficiently large $n$ it must be the case that

$$
\left|\varepsilon\left(t_{n}\right)\right|<2 M
$$

From (3.5) it now follows that $\alpha\left(t_{n}\right)=0$. This contradicts the definition of $\mathbf{H}$.

Since $\mathrm{H}$ is a finite set, there exists a $T_{1}>0$ and when $t \geqslant T_{1}, \alpha(t)=0$. This yields

$$
|\varepsilon(t)|<2 M
$$

From (3.20) and (3.29) we finally have

$$
|\hat{A} y(t)-\hat{B} u(t)|<2 M
$$

which establishes part (ii) of the theorem.

\section{Conclusion}

In this paper we have carried out convergence analysis of an adaptive GPP control algorithm. Our analysis shows that with some modifications of the self-tuning GPP algorithm given in Lelic and Zarrop (1987) global convergence of the adaptive control 
algorithm can be established. A technique used here is to fix the nonlinear parameters of the control law to some prespecified constant. Then a linear controller structure can be obtained. This enabled us to use the standard adaptive control analysis techniques in our paper.

\section{ACKNOWLEDGMENTS}

The first author's work was supported by the Postdoctoral Fellowship of the Royal Norwegian Council for Scientific and Industrial Research (NTNF) and the National Natural Science Foundation of P.R. China.

\section{REFERENCES}

Clarke, D. W., Mohtadi, C. and TuFF, P. S. (1987). Generalized predictive control. Automatica, 23, 137-160.

De Keyser, R. M. C., and Van CAuwengerghe, A. R. (1985). Extended prediction self-adaptive control. Proc. 7th IFAC Symp. on Identification and System Parameter Estimation, pp. 1255-1260.

Goodwin, G. C. and Sin, K. S. (1981). Adaptive control of nonminimum phase system. IEEE Trans. Automatic Control, 26, 478-483.

Goodwin, G. C. and Sin, K. S. (1984). Adaptive Filtering, Prediction, and Control (Prentice-Hall Englewood Cliffs, NJ).

Gu, XING YUAN and WANG Wei (1988). A stable discrete-time multivariable adaptive control in the presence of unmodeled dynamics. Proc. 8th IF AC Symp. on Identification and System Parameter Estimation, pp. 265-270.

GU, XING YUAN and WANG WEI (1989). On the stability of a self-tuning controller in the presence of bounded disturbances, IEEE Trans. Automatic Control, 34, 211-214.

LeLIC, M. A. and ZARroP, M. B. (1987). Generalized pole-placement self-tuning controller Part 1. Basic algorithm. Int. J. Control, 46, 548-568.

ORTEGA, R. and SANCHEZ, G. (1989). Globally convergent multistep receding horizon adaptive controller. Int. J. Control, 49, 1655-1664.

YDSTIE, B. (1984). Extended horizon adaptive control. Proc. 9th IF AC World Congress, pp. 133137. 